The Chittagong Univ. J. B. Sci., Vol. 3(1 \&2):pp.01-09, 2008.

\title{
IN VITRO MICROPROPAGATION OF RAUVOLFIA SERPENTINA (L.) BENTH THROUGH INDUCTION OF DIRECT AND INDIRECT ORGANOGENESIS
}

\author{
S. K. BHADRA, T. K. BHOWMIK AND P. SINGH * \\ Department of Botany, University of Chittagong, Chittagong - 4331, Bangladesh
}

\begin{abstract}
Leaf and nodal segments of two months old field grown seedlings of Rauvolfia serpentina (L.) Benth were aseptically cultured on agar solidified MS medium supplemented with various combinations and concentrations of auxins (NAA, IAA, 2,4-D and picloram) and cytokinins (BAP and $\mathrm{Kn}$ ). The nodal segments produced highest number of multiple shoot buds (5.85/explant) on MS medium supplemented with $2.0 \mathrm{mgl}^{-1} \mathrm{BAP}+0.2 \mathrm{mgl}^{-1} \mathrm{NAA}$ or $2.0 \mathrm{mgl}^{-1} \mathrm{BAP}+0.1 \mathrm{mgl}^{-1}$ IAA. Whereas nodal segment produced callus tissue of different nature on MS medium supplemented with $1.5 \mathrm{mgl}^{-1} \mathrm{BAP}+0.5 \mathrm{mgl}^{-1} \mathrm{IAA}+1.5 \mathrm{mgl}^{-1}$ 2,4-D; 3.0 $\mathrm{mgl}^{-1} \mathrm{BAP}+1.0 \mathrm{mgl}^{-1} \mathrm{NAA}+1.5 \mathrm{mgl}^{-1} \mathrm{Kn}$ and $0.1 \mathrm{mgl}^{-1} \mathrm{Pic}+1.0 \mathrm{mgl}^{-1} \mathrm{Kn}$. The callus tissue of light green and nodular nature on further subculture in a wide range of plant growth regulators (PGRs) supplemented media, differentiated into multiple shoot buds that underwent rapid elongation on $2.0 \mathrm{mg} / \mathrm{l} \mathrm{BAP}$ and 0.2 mg/l NAA supplemented media. The elongated shoot buds on further subculture in rooting media produced strong and stout roots. Half strength MS with $1.5 \%$ (w/v) sucrose was most effective for enhancing rooting. Finally those plantlets were acclimatized in field. Thus a protocol was established for rapid micropropagation of this medicinal plant through induction of direct and indirect organogenesis from nodal explant.
\end{abstract}

Key words: Micropropagation, Rauvolfia Serpentina, Organogenesis

\section{INTRODUCTION}

$R$. serpentina (L.) Benth commonly known as 'Sarpagandha' is a small erect shrub with pale bark. Roots of this plant are used for medicinal purposes as they contain some important alkaloids such as reserpine, deserpidine, ajmaline, ajmalinine, serpentine and serpentinine. The roots are used as a valuable remedy for high blood pressure, insomnia, anxiety, insanity, epilepsy and other disorders of the central nervous system (Kirtikar and Basu 1975). This plant species is becoming rare in

\footnotetext{
* Corresponding author. 
BHADRA ET AL.

Bangladesh and, therefore, needs to be propagated rapidly to meet up our medicinal demand and also for conservation purposes. Tissue culture technique has been proved very efficient in rapid propagation of many medicinal plants (Erdei et al. 1981, Tsay and Huang 1989, Fay 1992, Sagare et al. 2000, Lakshmi and Mythili 2003). This research study was undertaken with a view to develop a reliable, reproducible and efficient protocol for in vitro propagation of this medicinal plant species.

\section{MATERIALS AND METHODS}

Two months old seedlings were collected from the nursery of BCSIR, Chittagong, and were established in garden pots of Botany Deparment of Chittagong University. Leaf and nodal segments of approximate $0.5-1.0 \mathrm{~cm}$ size were used as explant. Surface sterilization of the leaves and nodes was done by dipping in $70 \%$ ethanol for $30 \mathrm{sec}$. and submerging them in $0.2 \%$ (w/v) $\mathrm{HgCl}_{2}$ solution for $10 \mathrm{~min}$. The segments were then washed three times with sterile distilled water and finally cultured in test tube $(2.5 \times 15 \mathrm{~cm})$ and conical flask (100 ml). MS basal medium (Murashige and Skoog 1962) supplemented with various combinations of plant growth regulators (PGRs), viz., auxins (NAA, IAA, 2,4-D and Picloram) and cytokinins (BAP and $\mathrm{Kn}$ ) at different concentrations were used for culture of the explants. All the media were made $0.8 \%(\mathrm{w} / \mathrm{v})$ agar solidified and $\mathrm{pH}$ of the media was adjusted to 5.8 prior to autoclaving for $20 \mathrm{~min}$. at $121^{\circ} \mathrm{C}$ under $1.9 \mathrm{~kg} / \mathrm{cm}^{2}$ pressure. Culture vessels with inoculated explants were maintained in a culture room under a regular cycle of 14h light and 10h dark at $25 \pm 2{ }^{0} \mathrm{C}$. The nodal segments underwent direct or indirect organogenesis in some of the media combinations giving rise to multiple shoot buds. The multiple shoot buds were further grown on elongation media and finally the elongated shoot buds were individually rooted on rooting media. The complete plantlets were finally transferred and established in outside natural environment through successive phases of acclimatization.

\section{RESULTS AND DISCUSSION}

Within 25 - $30 \mathrm{~d}$ of culture both leaf and nodal segments gave different responses to different PGRs combinations and the type of response was dependent on both explant and PGRs. Nodal segments underwent direct organogenesis producing multiple shoot buds when cultured on (i) $2.0 \mathrm{mg} / \mathrm{l} \mathrm{BAP}+2.0 \mathrm{mg} / \mathrm{l}$ NAA (ii) $1.0 \mathrm{mg} / \mathrm{l} \mathrm{NAA}+0.5 \mathrm{mg} / \mathrm{l} \mathrm{BAP}$ (iii) $2.0 \mathrm{mg} / \mathrm{l} \mathrm{BAP}+0.2 \mathrm{mg} / \mathrm{l} \mathrm{NAA}$ (iv) $2.0 \mathrm{mg} / \mathrm{l} \mathrm{BAP}(\mathrm{v}) 1.0 \mathrm{mg} / \mathrm{Kn}+3.0 \mathrm{mg} / \mathrm{l} \mathrm{BAP}+1.0 \mathrm{mg} / \mathrm{l} \mathrm{NAA}$ and (vi) $0.1 \mathrm{mg} / \mathrm{l}$ 


\section{IN VITRO MICROPROPAGATION OF RAUVOLFIA SERPENTINA}

IAA $+2.0 \mathrm{mg} / \mathrm{l}$ BAP supplemented media (Table 1). The highest number of multiple shoot buds was, however, produced on the medium fortified with (i) 0.1 $\mathrm{mg} / \mathrm{l} \mathrm{IAA}+2.0 \mathrm{mg} / \mathrm{l} \mathrm{BAP}$ and (ii) $2.0 \mathrm{mg} / \mathrm{l} \mathrm{BAP}+0.2 \mathrm{mg} / \mathrm{l} \mathrm{NAA}$ (Fig. 1a). This finding indicates that BAP supplemented media was more effective in induction of multiple shoot buds. Mao et al. (1995) reported that BAP was superior to other cytokinins for inducing multiple shoots in Clerodendrum colebrookianum. Induction of multiple shoot buds directly from nodal explant has also been reported in a wide range of plant species (Murashige 1974, Miller et al. 1991). Nodal segments, however, produced callus tissue on a range of PGR supplemented media. Green and granular callus was produced on $3.0 \mathrm{mg} / \mathrm{l} \mathrm{BAP}+$ $1.0 \mathrm{mg} / \mathrm{l} \mathrm{NAA}+1.5 \mathrm{mg} / \mathrm{l} \mathrm{Kn}$ supplemented medium (Fig. 1b). After 2 - 3 subcultures on the same medium, these green callii multiplied along with differentiation of shoot buds. Thus indirect organogenesis took place in the course of culture. This finding indicates that combination of BAP, Kn and NAA was effective for inducing indirect organogenesis. Sarker et al. (1996) reported that BA and NAA combination was effective for shoot bud differentiation from callus of $R$. serpentina. Nodal segments produced white and nodular callus on the medium fortified with $1.5 \mathrm{mg} / \mathrm{l} \mathrm{BAP}, 0.5 \mathrm{mg} / \mathrm{l} \mathrm{IAA}$ and $1.5 \mathrm{mg} / \mathrm{l}$ 2,4-D. Whereas, loose and friable callus was produced on $0.1 \mathrm{mg} / \mathrm{l} \mathrm{Pic}$ and $1.0 \mathrm{mg} / \mathrm{l} \mathrm{Kn}$ containing medium (Table $2 \&$ Fig. 1c). After 2 - 3 subcultures on the same media, the white callus differentiated producing rhizoids.

TABLE 1: MULTIPLE SHOOT BUDS DEVELOPMENT FROM NODAL SEGMENTS OF $R$. SERPENTINA ON MS MEDIUM SUPPLEMENTED WITH DIFFERENT PGR COMBINATIONS.

\begin{tabular}{|c|c|c|c|}
\hline PGR combination & Explants* & $\begin{array}{l}\text { Time }(\mathrm{d}) \text { required } \\
\text { for multiple shoot } \\
\text { buds initiation }\end{array}$ & $\begin{array}{c}\text { Average number } \\
\text { of multiple shoot } \\
\text { buds/explant }\end{array}$ \\
\hline $2.0 \mathrm{mgl}^{-1} \mathrm{BAP}+2.0 \mathrm{mgl}^{-1} \mathrm{NAA}$ & $\begin{array}{l}\text { NS } \\
\text { LS }\end{array}$ & $\begin{array}{c}15-25 \\
-\end{array}$ & $\begin{array}{c}2.71 \\
-\end{array}$ \\
\hline $1.0 \mathrm{mgl}^{-1} \mathrm{NAA}+0.5 \mathrm{mgl}^{-1} \mathrm{BAP}$ & $\begin{array}{l}\text { NS } \\
\text { LS }\end{array}$ & $\begin{array}{c}20-25 \\
-\end{array}$ & $\begin{array}{c}1.00 \\
-\end{array}$ \\
\hline $2.0 \mathrm{mgl}^{-1} \mathrm{BAP}+1.0 \mathrm{mgl}^{-1} \mathrm{IAA}$ & $\begin{array}{l}\text { NS } \\
\text { LS }\end{array}$ & - & - \\
\hline $2.0 \mathrm{mgl}^{-1} \mathrm{BAP}+0.2 \mathrm{mgl}^{-1} \mathrm{NAA}$ & $\begin{array}{l}\text { NS } \\
\text { LS }\end{array}$ & $\begin{array}{c}15-20 \\
-\end{array}$ & $\begin{array}{c}5.85 \\
-\end{array}$ \\
\hline
\end{tabular}


BHADRA ET AL.

\begin{tabular}{cccc}
\hline $2.0 \mathrm{mgl}^{-1} \mathrm{BAP}$ & $\mathrm{NS}$ & $15-20$ & 2.28 \\
$3.0 \mathrm{mgl}^{-1} \mathrm{BAP}+1.0 \mathrm{mgl}^{-1} \mathrm{NAA}+1.0$ & $\mathrm{LS}$ & - & - \\
$\mathrm{mgl}^{-1} \mathrm{Kn}$ & $\mathrm{NS}$ & $20-25$ & 3.28 \\
& & - & - \\
\multirow{2}{*}{$1.0 \mathrm{mgl}^{-1} \mathrm{BAP}+0.1 \mathrm{mgl}^{-1} \mathrm{IBA}$} & $\mathrm{NS}$ & - & - \\
& LS & - & - \\
$1.0 \mathrm{mgl}^{-1} \mathrm{BAP}+0.1 \mathrm{mgl}^{-1} \mathrm{NAA}$ & $\mathrm{NS}$ & - & - \\
& LS & - & - \\
$2.0 \mathrm{mgl}^{-1} \mathrm{BAP}+0.1 \mathrm{mgl}^{-1} \mathrm{IAA}$ & $\mathrm{NS}$ & $15-20$ & - \\
$0.1 \mathrm{mgl}^{-1} \mathrm{Pic}+1.0 \mathrm{mgl}^{-1} \mathrm{BAP}$ & $\mathrm{LS}$ & - & - \\
& $\mathrm{NS}$ & - & - \\
\hline
\end{tabular}

$* \mathrm{NS}=$ Nodal segment, $\mathrm{LS}=$ Leaf segment

TABLE 2: CALLUS INDUCTION FROM NODAL AND LEAF SEGMENTS OF $R$. SERPENTINA ON MS MEDIUM SUPPLEMENTED WITH DIFFERENT PGR COMBINATIONS.

\begin{tabular}{|c|c|c|c|c|c|c|}
\hline PGR combination & Explants* & $\begin{array}{c}\text { No. of } \\
\text { explants } \\
\text { used }\end{array}$ & $\begin{array}{c}\text { No. of } \\
\text { explants } \\
\text { responsed }\end{array}$ & $\begin{array}{c}\% \text { of } \\
\text { response }\end{array}$ & $\begin{array}{c}\text { Types of } \\
\text { responses }\end{array}$ & $\begin{array}{l}\text { Time }(\mathrm{d}) \\
\text { required for } \\
\text { callus } \\
\text { induction }\end{array}$ \\
\hline $15 \mathrm{~mol}^{-1} \mathrm{BAP}+05 \mathrm{~mol}^{-1}$ & NS & 10 & 8 & 80 & White & $15-20$ \\
\hline IAA $+1.5 \mathrm{mgl}^{-1} 2,4-\mathrm{D}$ & LS & 10 & 3 & 30 & $\begin{array}{l}\text { granular } \\
\text { callus }\end{array}$ & $15-20$ \\
\hline $2.0 \mathrm{mgl}^{-1} \mathrm{BAP}+0.2 \mathrm{mgl}^{-1}$ & NS & 10 & - & - & - & - \\
\hline IAA & LS & 10 & - & - & - & - \\
\hline $0.1 \mathrm{mgl}^{-1} \mathrm{BAP}+0.1 \mathrm{mgl}^{-1}$ & NS & 10 & - & - & White and & - \\
\hline Pic & LS & 10 & 2 & 20 & $\begin{array}{l}\text { compact } \\
\text { callus }\end{array}$ & $15-20$ \\
\hline $2.0 \mathrm{mgl}^{-1} \mathrm{BAP}+0.5 \mathrm{mgl}^{-1}$ & NS & 10 & - & - & White and & - \\
\hline Pic & LS & 10 & 2 & 20 & compact & $20-25$ \\
\hline $3.0 \mathrm{mgl}^{-1} \mathrm{BAP}+1.0 \mathrm{mgl}^{-1}$ & NS & 10 & 6 & 60 & Green & $20-25$ \\
\hline $\mathrm{NAA}+1.5 \mathrm{mgl}^{-1} \mathrm{Kn}$ & LS & 10 & - & - & $\begin{array}{l}\text { granular } \\
\text { callus }\end{array}$ & - \\
\hline $0.1 \mathrm{mgl}^{-1} \mathrm{Pic}+1.0 \mathrm{mgl}^{-1}$ & NS & 10 & 4 & 40 & White, & $20-25$ \\
\hline $\mathrm{Kn}$ & LS & 10 & 1 & 10 & Soft & $20-25$ \\
\hline
\end{tabular}

$* \mathrm{NS}=$ Nodal segment, LS $=$ Leaf segment, '-' = indicates no response. 


\section{IN VITRO MICROPROPAGATION OF RAUVOLFIA SERPENTINA}

For induction of embryogenesis/ organogenesis the callus that was induced from leaf and nodal segments was further cultured on a broad spectrum of PGRs supplemented media (Table 3). White callus failed to undergo any kind of differentiation and finally died. When green and granular callus was cultured on the medium supplemented with $3.0 \mathrm{mg} / \mathrm{l} \mathrm{BAP}+1.0 \mathrm{mg} / \mathrm{l} \mathrm{Kn}+1.0 \mathrm{mg} / \mathrm{l} \mathrm{NAA}$, it multiplied and differentiated into shoot buds (Fig. 1d). This observation suggests that only the green and nodular callus had morphogenic competence for regeneration. Similar result was reported by Sarker et al. (1996). In order to induce rapid elongation, multiple shoot buds originated either through direct organogenesis or through the intervention of callus were grown on different PGRs supplemented media (Table 4). MS Medium fortified with $2.0 \mathrm{mg} / \mathrm{l} \mathrm{BAP}+0.2$ mg/l NAA was proved efficient for rapid elongation (Fig. 1e). Ahmed et al. (2002) also noted similar findings in this plant species. On elongation media shoot buds did not produce any root.
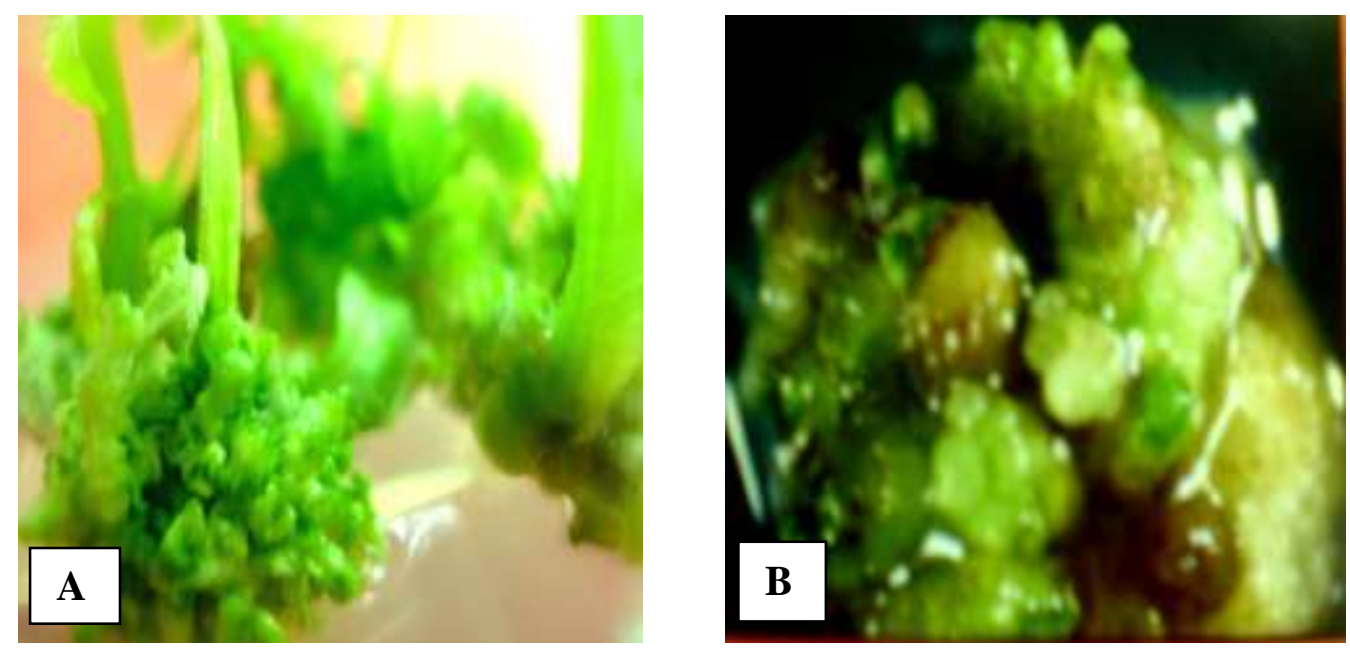

FIGURE 1 PAGE 6 CONTINUE 

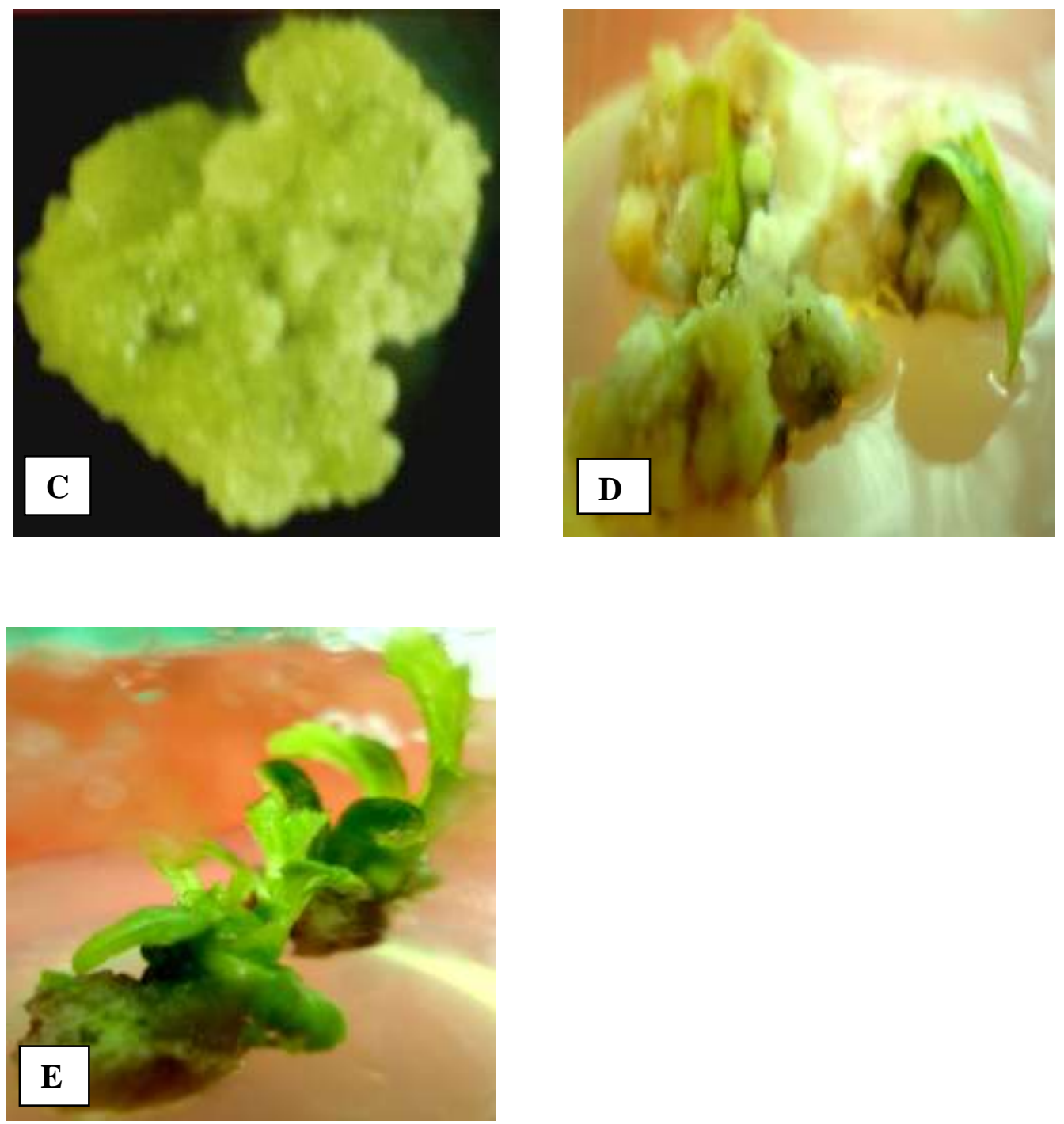

FIGURE 1 A: MULTIPLE SHOOT BUDS DEVELOPED FROM NODAL SEGMENT OF RAUVOLFIA SERPENTINA; B, COMPACT GRANULAR AND GREEN TISSUE DEVELOPED FROM NODAL SEGMENT; C, LOOSE AND FRIABLE TISSUE INDUCED IN NODAL SEGMENTS; D, GREEN AND GRANULAR TISSUE UNDERGOING DIFFERENTIATION TO FORM SHOOT BUDS; E, SHOOT BUDS UNDERGOING RAPID ELONGATION IN ELONGATION MEDIUM. 
IN VITRO MICROPROPAGATION OF RAUVOLFIA SERPENTINA

TABLE 3 : RESULTS OF FURTHER CULTURE OF INDUCED CALLUS OF R. SERPENTINA ON MS MEDIUM SUPPLEMENTED WITH DIFFERENT PGR COMBINATIONS.

\begin{tabular}{|c|c|c|}
\hline PGR combination & $\begin{array}{l}\text { Initial colour of } \\
\text { callus tissue }\end{array}$ & $\begin{array}{l}\text { Nature of response after } 45 \mathrm{~d} \text { of } \\
\text { culture on the medium }\end{array}$ \\
\hline $1.5 \mathrm{mgl}^{-1} \mathrm{BAP}+0.2 \mathrm{mgl}^{-1} \mathrm{NAA}$ & White & $\begin{array}{l}\text { Multiplied them became brownish } \\
\text { and death }\end{array}$ \\
\hline $1.0 \mathrm{mgl}^{-1} \mathrm{BAP}+0.1 \mathrm{mgl}^{-1} \mathrm{NAA}$ & White & Multiplied and then died \\
\hline $2.0 \mathrm{mgl}^{-1} \mathrm{BAP}+0.1 \mathrm{mgl}^{-1} \mathrm{NAA}$ & Green & $\begin{array}{l}\text { Multiplied, become light green and } \\
\text { produced granular callus }\end{array}$ \\
\hline $2.0 \mathrm{mgl}^{-1} \mathrm{BAP}+2.0 \mathrm{mgl}^{-1} \mathrm{NAA}$ & White & Multiplied and then died \\
\hline $2.0 \mathrm{mgl}^{-1} \mathrm{BAP}+1.0 \mathrm{mgl}^{-1} \mathrm{IAA}$ & White & $\begin{array}{c}\text { No multiplication occurred, became } \\
\text { brown and finally died }\end{array}$ \\
\hline $1.0 \mathrm{mgl}^{-1} \mathrm{BAP}+2.0 \mathrm{mgl}^{-1} \mathrm{Kn}$ & White & $\begin{array}{c}\text { No multiplication occurred, became } \\
\text { brown and finally died }\end{array}$ \\
\hline $\begin{array}{c}3.0 \mathrm{mgl}^{-1} \mathrm{BAP}+1.0 \mathrm{mgl}^{-1} \mathrm{NAA}+1.0 \mathrm{mgl}^{-1} \\
\mathrm{Kn}\end{array}$ & Green & $\begin{array}{l}\text { Multiplied along with organogenesis } \\
\text { producing shoot buds }\end{array}$ \\
\hline
\end{tabular}

TABLE 4 : ELONGATION OF MULTIPLE SHOOT BUDS DEVELOPED FROM NODAL SEGMENTS OF $R$. SERPENTINA ON MS MEDIUM SUPPLEMENTED WITH DIFFERENT PGR COMBINATIONS.

\begin{tabular}{|c|c|c|c|}
\hline PGR combination & $\begin{array}{c}\text { Average initial } \\
\text { length }(\mathrm{cm}) * \text { of } \\
\text { individual multiple } \\
\text { shoot bud }\end{array}$ & $\begin{array}{l}\text { Average length }(\mathrm{cm}) \text { of } \\
\text { individual multiple } \\
\text { shoot bud after } 45 \mathrm{~d} \text { of } \\
\text { culture on elongation } \\
\text { medium }\end{array}$ & $\begin{array}{l}\text { Increased in length } \\
(\mathrm{cm}) \text { of shoot bud } \\
\text { within } 45 \mathrm{~d} \text { of } \\
\text { culture on } \\
\text { elongation medium }\end{array}$ \\
\hline $2.0 \mathrm{mgl}^{-1} \mathrm{BAP}+2.0 \mathrm{mgl}^{-1} \mathrm{NAA}$ & 1.06 & 2.43 & 1.37 \\
\hline $\begin{array}{c}0.2 \mathrm{mgl}^{-1} \mathrm{NAA}+2.0 \mathrm{mgl}^{-1} \\
\mathrm{BAP}\end{array}$ & 1.16 & 3.96 & 2.80 \\
\hline $\begin{array}{c}3.0 \mathrm{mgl}^{-1} \mathrm{BAP}+1.0 \mathrm{mgl}^{-1} \mathrm{NAA} \\
+1.0 \mathrm{mgl}^{-1} \mathrm{Kn}\end{array}$ & 1.29 & 2.98 & 1.69 \\
\hline $0.1 \mathrm{mgl}^{-1} \mathrm{IAA}+2.0 \mathrm{mgl}^{-1} \mathrm{BAP}$ & 1.25 & 2.84 & 1.59 \\
\hline
\end{tabular}

* Only the length of shoot system. 
For induction of strong and stout root system the elongated shoots were individually grown on four different rooting media, viz., (i) half strength MS + $1.5 \%(\mathrm{w} / \mathrm{v})$ sucrose (ii) half strength $\mathrm{MS}+1.5 \%(\mathrm{w} / \mathrm{v})$ sucrose $+0.5 \mathrm{mg} / \mathrm{l} \mathrm{IAA}+$ $0.5 \mathrm{mg} / \mathrm{l} \mathrm{IBA}$ (iii) half strength MS $+1.5 \%(\mathrm{w} / \mathrm{v})$ sucrose $+0.5 \mathrm{mg} / \mathrm{l}$ NAA and (iv) full strength $\mathrm{MS}+3 \%(\mathrm{w} / \mathrm{v})$ sucrose $+0.5 \mathrm{mg} / \mathrm{l}$ IAA. Half strength MS with $1.5 \%(\mathrm{w} / \mathrm{v})$ sucrose was found most effective for rooting. It proved, the auxin free medium was best for rooting. Such result was also reported by Azad et al. (1999). Well developed plantlets were then transferred to outside under natural environment through sequential phases of acclimatization. The protocol thus developed for mass production of seedlings of $R$. serpentina can be used reliably for conservation of this important medicinal plant species of Bangladesh.

\section{REFERENCES}

AHMED, S., AMIN, M.N., AZAD, M.A.K. AND MOSADDIK, M.A. 2002. Micropropagation and plant regeneration of Rauvolfia serpentina by tissue culture technique. Pakistan J. Biol. Sci. 5(1): 75-79.

AZAD, M.A.K., AMIN, M.N. AND BEGUM, F. 1999. In vitro rapid regeneration of plantlets from cotyledon explant of Adhatoda vasica Nees. Plant Tissue Cult. 9(2): 121-126.

ERDEI, I., KISS, Z. AND MALIGA, P. 1981. Rapid clonal multiplication of Digitalis lanata in tissue culture. Plant Cell Reports. 1: 34-35.

FAY, M.F. 1992. Conservation of rare and endangered plants using in vitro methods. In vitro Cellular and Development Biology. 28: 1-4.

KIRTIKAR, K.R. AND BASU, B.D. 1975. Plumbaginaceae in Indian Medicinal Plants, $2^{\text {nd }}$ edition,. Vol. II. Published by Bishen Singh, Mahendra Pal Singh, Dehra Dun, India. PP. 1465-1470.

LAKSHMI, M. AND MYTHILI, S. 2003. Somalic embryogenesis and regeneration from callus cultures of Kaempferia galang - a medicinal plant. J. Medicinal and Aromatic Plant Science. 25: 947-951.

MAO, A.H., WETTEN, A., FAY, M. AND CALIGARI, P.D.S. 1995. In vitro propagation of Clerodendrum colebrookianum a potential natural antihypertension medicinal plant. Plant Cell Rep. 14: 493-496.

MILLER, R.M., KAUL, V., HUTCHINSON, J.F. AND RICHARDS, D. 1991. Adventitious shoot regeneration in carnation (Dianthus caryophyllus L.) from axillary bud explants. Annals Bot. 67(1): 35-42.

MURASHIGE, T. 1974. Plant propagation through tissue culture. Ann. Rev. Plant Physiol. 25: 135-166. 
MURASHIGE, T. AND SKOOG, F. 1962. A revised medium for rapid growth and bioassay with tobacco tissue culture. Physiologia Plantarum 15: 473-497.

SAGARE, A.P., LEE, Y.L., LIN, T.C., CHEN, C.C. AND TSAY, H.S. 2000. Cytokinin induced somatic embryogenesis and plant regeneration in Corydalis yanhusuo (Fumariaccae) a medicinal plant. Plant Science 160: 139-147.

SARKER, K.P., ISLAM, R., ISLAM, A., HOQUE, A. AND JOARDER, O.I. 1996. Plant regeneration of Rauvolfia serpentina by organogenesis from callus cultures. Plant Tissue Cult. 6 (1): 63-65.

TSAY, H.S. AND HUANG, H.L. 1989. Somatic embryo formation and germination from immature embryo-derived suspension cultured cells of Angelica sinensis (oliv.) Diels. Plant Cell Reports. 17: 670- 674.

Manuscript received on 19.5.07; Accepted on 20. 9. 09

The Chittagong University Journal of Biological Sciences, Vol. 3( 1 \& 2): pp.01-09, 2008 